\title{
EP-123
}

\section{Intrathoracic calculous cholecystitis due to delayed iatrogenic diaphragmatic hernia}

\author{
Jae Do YANG*, Mi Rin LEE, Sung Woo AHN, Hee Chul YU
}

Department of Surgery, Jeonbuk National University Hospital, Jeonju, Korea

Introduction: Iatrogenic diaphragmatic hernias is rare complications of abdominal or thoracic surgery. Depending on the location and size of the defect retroperitoneal or intra-abdominal organs and tissues can prolapse into thoracic cavity due to the negative intra-thoracic pressure. This can cause risk of obstruction or strangulation of abdominal contents, and may ischemia and perforation.

Methods: We present a case of delayed presentation of a patient with iatrogenic diaphragmatic herniation of liver and gallbladder with stones into the thorax.

Results: A 62-year-old female was visited our hospital for complaining of right sided thoracic back pain during 3 month. The pain had not been treated with analgesics. Three years ago, she has been diagnosed right diaphragmatic metastatic nodule from ovarian cancer. She was operated excisional biopsy of intrathoracic lymph nodes and parietal pleural by VATs (video-assisted thoracic surgery). A computed tomography-scan showed intrathoracic displacement of her liver and chronic cholecystitis with multiple stones. The patients underwent laparotomy via an abdominal approach with subcostal incision. Intraoperative findings confirmed that right diaphragmatic focal defect and liver and gallbladder herniated into the thoracic cavity. The organs were placed back into the thoracic cavity. A cholecystectomy was performed, and the defect in the diaphragm measuring $13 \mathrm{~cm} \times 10 \mathrm{~cm}$, was repaired with a Gortex graft.

Conclusions: Postoperative recovery was uneventful. The patient was discharged seven days after the surgery without any problems. 